\title{
Activation of the T1 Neuronal Circuit is Necessary and Sufficient to Induce Sexually Dimorphic Mating Behavior in Drosophila melanogaster
}

\author{
David S. Ronderos ${ }^{2}$ and Dean P. Smith ${ }^{1,2}$ \\ Departments of ${ }^{1}$ Pharmacology and ${ }^{2}$ Neuroscience, University of Texas Southwestern Medical Center, Dallas, Texas 75390-9111
}

The molecular and cellular events mediating complex behaviors in animals are largely unknown. Elucidating the circuits underlying behaviors in simple model systems may shed light on how these circuits function. In drosophila, courtship behavior provides a tractable model for studying the underlying basis of innate behavior. The male-specific pheromone 11-cis-vaccenyl acetate (cVA) modulates courtship behavior and is detected by T1 neurons, located on the antenna of male and female flies. The T1 neurons express the odorant receptor Or67d and are exquisitely tuned to $\mathrm{cVA}$ pheromone. However, cVA-induced changes in mating behavior have also been reported upon manipulation of olfactory neurons expressing odorant receptor Or65a. These findings raise the issue of whether multiple olfactorydriven circuits underlie cVA-induced behavioral responses and what role these circuits play in behavior. Here, we engineered flies in which the Or67d circuit is specifically activated in the absence of cVA to determine the role of this circuit in behavior. We created transgenic flies that express a dominant-active, pheromone-independent variant of the extracellular pheromone receptor, LUSH. We found that, similar to the behaviors elicited by cVA, engineered male flies have dramatically reduced courtship, whereas engineered females showed enhanced courtship. cVA exposure did not enhance the dominant LUSH-triggered effects on behavior in the engineered flies. Finally, we show the effects of both cVA and dominant LUSH on courtship are reversed by genetically removing Or67d. These findings demonstrate that the T1/0r67d circuit is necessary and sufficient to mediate sexually dimorphic courtship behaviors.

\section{Introduction}

In most animals, behaviors are elicited in response to sensory inputs. One well characterized set of stereotypical behavior patterns occurs during courtship in the fruit fly Drosophila melanogaster. Males actively court females through a series of ritual behaviors that ultimately conclude in copulation (for review, see Hall, 1994; Greenspan and Ferveur, 2000; Manoli et al., 2006; Dickson, 2008). Progression through courtship requires interactions between partners that are mediated through the visual, auditory, tactile, olfactory, and gustatory senses (Amrein, 2004; Dickson, 2008). Understanding how these inputs are detected and processed will shed light on the neuronal circuits that elicit a stereotypical behavioral output in a simple model system (Manoli et al., 2006).

One volatile cue known to modulate courtship behavior is the male-specific pheromone, 11-cis-vaccenyl acetate (cVA) (for review, see Ha and Smith, 2009; Ronderos and Smith, 2009). This pheromone is known to activate male and female T1 olfactory

Received Sept. 27, 2009; revised Dec. 1, 2009; accepted Dec. 10, 2009

This work was supported by National Institutes of Health/National Institute on Deafness and Other Communication Disorders Grant R21 DC009880. We thank Barry Dickson for the Or67 $d^{\text {GAL4 }}$ flies, and Jonathan Terman and Robin Hiesinger for critical review of this manuscript.

Correspondence should be addressed to Dean P. Smith, Departments of Pharmacology and Neuroscience, University of Texas Southwestern Medical Center, 5323 Harry Hines Boulevard, Dallas, TX 75390. E-mail: dean.smith@utsouthwestern.edu.

DOI:10.1523/JNEUROSCI.4819-09.2010

Copyright $\odot 2010$ the authors $\quad 0270-6474 / 10 / 302595-05 \$ 15.00 / 0$ neurons that express the odorant receptor Or67d (Clyne et al., 1997; Xu et al., 2005; Ha and Smith, 2006; Kurtovic et al., 2007). cVA binds directly to the extracellular binding protein LUSH, producing an activated conformation in LUSH (Laughlin et al., 2008). cVA-activated LUSH is a specific ligand for T1 neurons through a neuronal receptor consisting of at least three components, including Or67d (Ha and Smith, 2006; Benton et al., 2007; Kurtovic, 2007; Jin et al., 2008). The demonstration that activated LUSH, not cVA, is the ligand for this receptor complex was highlighted by finding a dominant-active mutant LUSH protein, LUSH $^{\text {D118A }}$, that activates T1 neurons in the absence of cVA but has no effect on any other class of olfactory neurons (Laughlin et al., 2008).

A role for the T1 neuronal circuit in courtship behavior came initially from the study of mutants lacking Or67d, Or67 $d^{\text {GAL4 }}$ (Kurtovic et al., 2007). Males lacking this receptor display increased courtship directed toward other males when compared with wild-type controls, and females lacking this receptor show prolonged latency to copulation (Kurtovic et al., 2007). However, these studies do not address whether activity in the Or67d olfactory neurons accounts for all or part of cVA-induced mating behavior. Recent studies reported that the odorant receptor Or65a, normally expressed by neurons in T2 trichoid sensilla [specifically, the at4 subtype of T2 (Couto et al., 2005)], can be activated by extremely high cVA concentrations when the receptor is misexpressed in basiconic neurons (van der Goes van Naters and Carlson, 2007). Furthermore, expressing tetanus 
toxin under control of the Or65a promoter disrupted cVA-induced suppression of courtship by virgin males, whereas expressing tetanus toxin under control of the Or67d promoter had no effect (Ejima et al., 2007). Therefore, it is possible that activation of multiple neuronal circuits, triggered through different odorant receptors, underlies cVA-induced behavior.

We set out to dissect the role of the T1 neural circuit on mating behavior. To determine the behavioral consequences of activating the Or67d circuit in the absence of other potentially contributing cVA circuits, we engineered and analyzed the behavior of flies in which the T1 circuit is constitutively activated in the absence of cVA pheromone.

\section{Materials and Methods}

Drosophila stocks. lush ${ }^{1}$ flies were described previously (Kim et al., 1998; Xu et al., 2005). Or67 $d^{\text {GAL4 }}$ mutants have the Or67 $d$ structural gene replaced by a Gal4 yeast transcription factor gene as described by Kurtovic et al. (2007). Transgenic lush ${ }^{D 118 A}$ flies were produced by injecting a mutated lush genomic rescuing construct (Kim et al., 1998) into lush ${ }^{1}$ mutants. Two independent transgenic insertion lines were studied. The wild-type control stock used was a $w^{1118}$ isogenic stock.

Generation of lush ${ }^{\mathrm{D} 118 \mathrm{~A}}$ construct. The lush ${ }^{\mathrm{D} 118 \mathrm{~A}}$ transgene was created by mutating a $4.8 \mathrm{~kb}$ genomic rescue construct previously shown to restore wild-type function to $l_{\text {ush }}{ }^{1}$ mutants (Kim et al., 1998). The codon for Aspartate 118 in LUSH is flanked by the restriction sites Mlul and PflM1, which are unique in the rescue construct. Overlapping PCR primers were designed to introduce the $\mathrm{D}$ to $\mathrm{A}$ change at codon 118 and were used to produce a PCR product containing the D118A change in a fragment spanning these restriction sites. This altered fragment was digested with Mlu1 and PflM1 and cloned into the Mlu1-PflM1 digested rescue construct. The successful introduction of the mutation was confirmed by sequencing.

Western blot and immunohistochemical analysis. Protein extracts from 30 antennas disrupted by probe sonication (Bransonic) were loaded per lane and proteins were separated by $10-20 \%$ Tris- $\mathrm{HCl}$ Ready Gel (BioRad Laboratories). The proteins were transferred to Optitran BA-S 83 (Whatman) $0.2 \mu \mathrm{m}$ nitrocellulose membrane using semidry blotting (Bio-Rad Laboratories), probed with anti-LUSH antibodies (Kim et al., 1998) and HRP-conjugated secondary antibodies (Jackson ImmunoResearch Laboratories), and detected with Supersignal (Thermo Scientific) as previously described (Kim et al., 1998). Immunohistochemical detection of LUSH antigen in $12-\mu \mathrm{m}$-thick Drosophila frozen tissue sections was performed with affinity purified anti-LUSH antiserum and detected with goat anti-rabbit 594 (Jackson ImmunoResearch Laboratories), and imaged using a Zeiss LSM 510 confocal microscope.

Single sensillum electrophysiology. Extracellular electrophysiological recordings were performed according to Ha and Smith (2006). Briefly, 2to 7-d-old flies were under a constant stream of charcoal-filtered air (36 $\mathrm{ml} / \mathrm{min} ; 22-25^{\circ} \mathrm{C}$ ) to prevent any potential environmental odors from affecting activity during these studies. Signals were amplified $100 \times$ (USB-IDAC System; Syntech) and fed into a computer via a 16-bit analog-digital converter and analyzed off-line with AUTOSPIKE software (USB-IDAC System; Syntech). Low cutoff filter setting was $200 \mathrm{~Hz}$, and the high cutoff was $3 \mathrm{kHz}$. Action potentials were recorded by inserting a glass electrode in the base of the sensillum. Signals were recorded for $10-15 \mathrm{~min}$ and averaged to determine spontaneous firing rates. All recordings were performed from separate sensilla with a maximum of two sensilla recorded from any single fly.
Mating behavior assays. Naive virgin males and females were kept in isolation from the time of eclosion until mating behavior assays were conducted. Single 3-7-d-old virgin male and female flies were placed in courtship chambers $(1.5-\mathrm{cm}$-diameter polystyrene wells containing $1 \%$ agarose in water covered with Whatman paper) and video recorded for 10 min. Courtship Index (CI) for all experiments was measured by dividing the time spent courting $\left(T_{\mathrm{C}}\right)$ by the total observation time $\left(T_{\mathrm{T}}\right)$ multiplied by $100 \%\left[\mathrm{CI}=\left(T_{\mathrm{C}} / T_{\mathrm{T}}\right) \times 100 \%\right]$. For these experiments, $T_{\mathrm{C}}$ is defined as the sum total time flies are engaged in any step of the courtship ritual [tracing/chasing, orienting, wing vibration/courtship song, abdominal curling, and copulation (Greenspan and Ferveur, 2000)]. For cVA experiments, $1 \mu \mathrm{l}$ of pure cVA was applied to the Whatman paper just before the assay. SE of measurement was calculated for each genotype and $p$ values were calculated using unpaired two-tailed Student's $t$ tests.

\section{Results}

Generation and characterization of transgenic flies expressing dominant-active lush $^{\text {D118A }}$

Previously, we showed that infusion of recombinant LUSH $^{\text {D118A }}$ into $\mathrm{T} 1$ sensilla induces robust activation of the T1 neurons independent of cVA (Laughlin et al., 2008). LUSH ${ }^{\text {D118A }}$ activates T1 neurons, but not non-T1 trichoid neurons (Laughlin et al., 2008). Therefore, by expressing LUSH $^{\text {D118A }}$ as a transgene under control of the lush promoter, we can isolate the behavioral effects of T1 circuit activation in the absence of cVA. We generated transgenic flies expressing $l u s h^{D 118 A}$ under control of the lush promoter and injected this construct into the $l u s h^{1}$ mutant background. In these engineered flies, wild-type LUSH protein is essentially replaced with dominantactive LUSH $^{\text {D118A }}$ (Fig. 1).

Protein expression levels of LUSH ${ }^{\mathrm{D} 118 \mathrm{~A}}$ in transgenic flies are similar to that of wild-type LUSH, as determined by Western blots of antennal extracts (Fig. 1). Consistent with its expression regulated by the lush promoter, immunohistochemical analysis reveals that $\mathrm{LUSH}^{\mathrm{D} 118 \mathrm{~A}}$ protein expression is restricted to the trichoid antennal regions and is detectable in the sensillum 


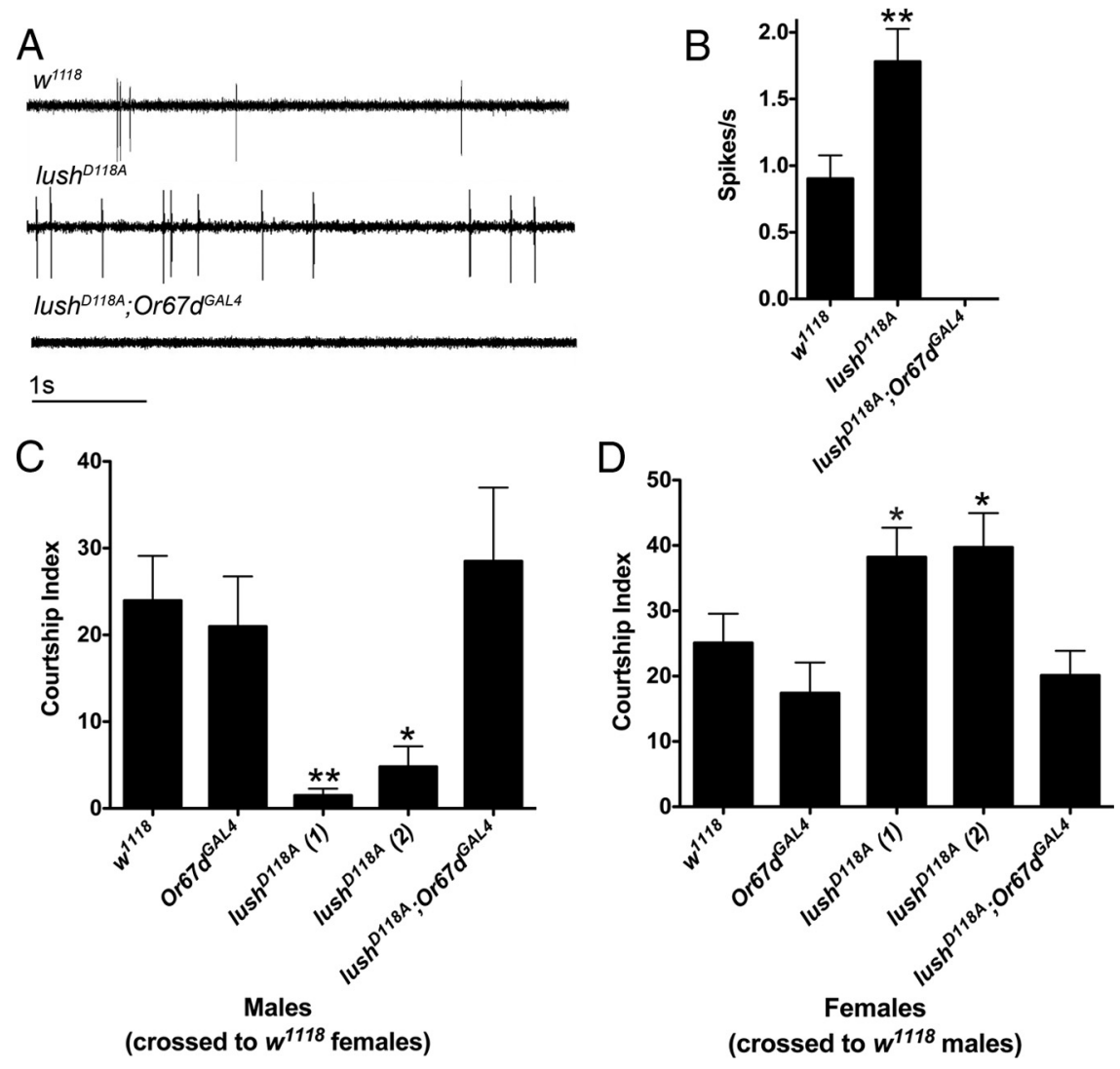

Figure 2. T1 neural activation is necessary and sufficient to induce sexually dimorphic courtship behavior. $\boldsymbol{A}$, Representative traces obtained from single sensillum electrophysiological recordings of T1 neurons of wild-type $\left(w^{1118}\right)$, I ush ${ }^{D 118 A}$ transgenic flies, or flies expressing /ush ${ }^{D 118 A}$ in an Or67d mutant background (lush ${ }^{D 118 A} ;$ Or67 $\left.d^{G A L 4}\right)$. B, Quantitation of spontaneous activity in T1 neurons from flies of genotypes described above. C, Male courtship index of wild-type, lush ${ }^{D 118 A}$, or lush ${ }^{D 118 A} ; \operatorname{Or}_{67 d^{6 A L 4}} \mathrm{flies}^{\text {(1) }}$ and (2) indicate two transgenic lines generated by independent $p$-element insertions. Males of the indicated genotypes were crossed to control $\left(w^{1118}\right)$ females. D, Female courtship index of wild-type, lush ${ }^{D 118 A}$, or lush ${ }^{D 118 A} ; \operatorname{Or} 67 d^{\text {GALA }}$ flies. Females of the indicated genotypes were crossed to control $\left(w^{1118}\right)$ males. Error bars represent SEM. Significant differences from the wild-type controls calculated using Student's $t$ test. For all bars, $n \geq 7 .{ }^{*} p<0.05,{ }^{* *} p<0.01$.

lymph in the distal portions of the trichoid sensilla in a pattern that is indistinguishable from wild-type LUSH in control flies (Fig. 1). Therefore, the transgene is expressed at wild-type levels and is secreted into the sensillum lymph. Electrophysiological analysis of the lush ${ }^{D 118 A}$ engineered flies revealed a significant increase in the basal firing rate of $\mathrm{T} 1$ neurons, averaging $200 \%$ above wild-type controls (Fig. $2 A, B$ ). The LUSH $^{\text {D118A }}$-induced activation of T1 neurons is mediated by the downstream receptor Or67d, as removal of Or67d abolishes the activating effect of lush $^{\text {D118A }}$ on T1 neurons (Fig. $2 A, B$ ). Similar to the results of direct infusion of recombinant $\mathrm{LUSH}^{\mathrm{D} 118 \mathrm{~A}}$ through a recording electrode (Laughlin et al., 2008), transgenic LUSH ${ }^{\text {D118A }}$ had no effect on the spontaneous action potential rates of other trichoid neurons (supplemental Fig. S3, available at www.jneurosci.org as supplemental material). Therefore, lush ${ }^{D 118 A}$ specifically activates $\mathrm{T} 1$ neurons in the absence of $\mathrm{cVA}$.

\section{lush ${ }^{D 118 A}$ phenocopies sexually dimorphic mating behavior elicited by cVA}

We tested the consequences of lush ${ }^{D 18 A}$ expression on the social behavior of the male and female transformants. Figure $2 C$ shows that males engineered to express lush ${ }^{D 118 A}$ have a dramatic reduction in courtship behavior toward wild-type females compared with their wild-type counterparts (see supplemental Fig. S1A,B, available at www.jneurosci.org as supplemental material). Two in- dependent transgenic lines showed a similar reduction in courting. These behavioral defects are not caused by general effects on locomotor behavior, as both transgenic lines showed similar activity levels compared with wild-type controls (supplemental Fig. S2, available at www.jneurosci.org as supplemental material). Furthermore, removal of the downstream receptor, Or67d, completely reverses the lush $h^{D 118 A}$-induced suppression of male courtship, indicating these behavioral changes are mediated through T1 neurons (Fig. 2C). Since cVA is only present on males, this effect on courtship behavior is consistent with LUSH ${ }^{\text {D118A }}$ activating T1 neurons that inhibit malemale courtship.

Females expressing lush ${ }^{D 18 A}$ also show altered social behaviors. Figure $2 D$ shows that transgenic females paired with wildtype males are significantly more likely to undertake courtship behavior than their wild-type counterparts. Both transgenic lines showed similar increases in female receptivity to courtship. Once again, these differences in female mating behavior are completely reversed by removal of the downstream receptor Or67d, suggesting that the effects of lush ${ }^{D 118 A}$ are mediated by T1 neurons (Fig. 2D). Together, these results show that lush ${ }^{D 118 A}$ expression mimics the effects of cVA exposure on both male and female courtship behavior.

\section{Sexually dimorphic cVA-triggered mating behaviors are mediated specifically through $\mathrm{T} 1$ neurons}

To test whether lush ${ }^{D 118 A}$ has the same effects on mating behavior as cVA, we determined the courtship index of wild-type controls both in the presence and absence of exogenous cVA. We find that addition of cVA to the mating chamber results in significant suppression of wild-type male courtship (Fig. 3). These responses are similar to the courtship index of lush ${ }^{D 118 A}$ males without cVA. Furthermore, addition of cVA to the mating chamber of lush ${ }^{D 118 A}$ males has no additional effect on their courtship (Fig. 3). We conclude that activation of T1 neurons by lush ${ }^{D 118 A}$ is sufficient to suppress male courtship and that further activation by cVA has no additional effect on mating behavior. Together, these results suggest that $\mathrm{T} 1$ neuron activation is sufficient to mediate cVA-induced suppression of male courtship behavior.

We next asked whether specific T1 neural circuit activation is necessary to induce behaviors normally associated with cVA exposure. We therefore tested mating behavior of $\mathrm{Or} 67 d^{\text {GALA }}$ males in the presence and absence of cVA. These flies are defective for expression of the T1 neuron odorant receptor Or67d (Kurtovic et al., 2007), an essential component of the receptor that is triggered by cVA-activated LUSH (Ha and Smith, 2006; Kurtovic et al., 2007; Laughlin et al., 2008). The courtship index of Or67 $d^{G A L 4}$ males paired with wild-type females was not significantly different from wild-type controls (Fig. 3). Therefore, these mutants mate normally with control females. However, addition of cVA to the mating chamber failed to suppress courtship in Or67d ${ }^{G A L 4}$ 


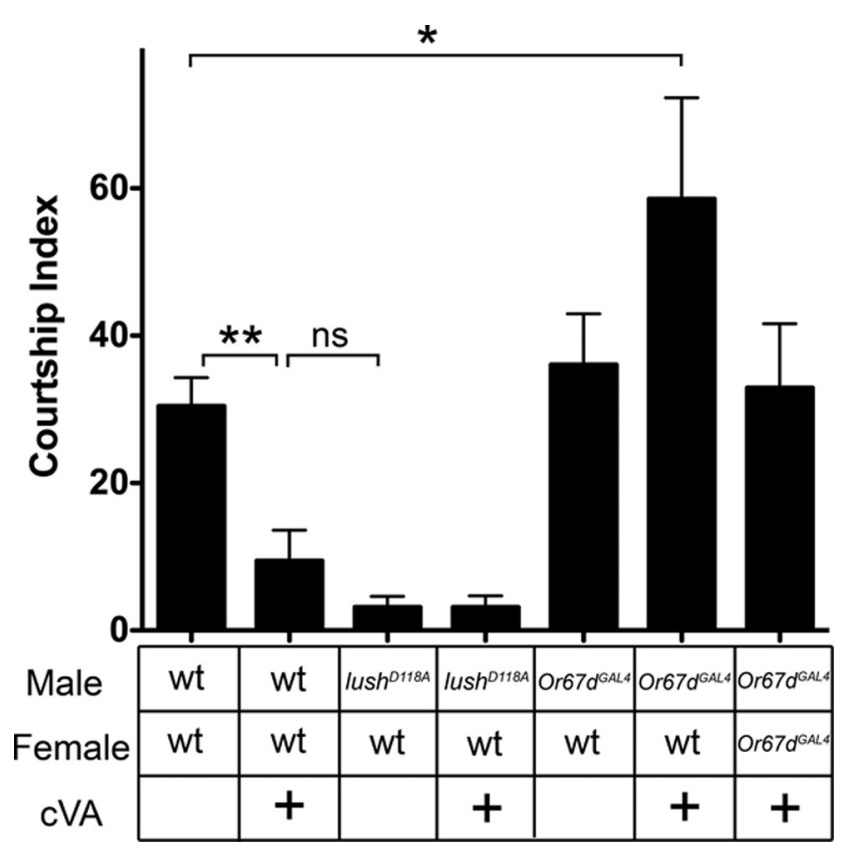

Figure 3. Sexually dimorphic CVA-triggered mating behaviors are mediated specifically by T1 neurons. CVA exposure suppresses male courtship behavior in wild-type (wt) males to a level indistinguishable from $/ u s h^{D 118 A}$ in the absence of CVA $(p>0.1)$. cVA exposure has no further suppression of courtship by lush ${ }^{D 118 A}$ males $(p>0.1)$. cVA fails to suppress courtship of Or67 $d^{G A L 4}$ mutant males and, when crossed to wild-type females, courtship index is enhanced due to the aphrodisiac effect of cVA on the female. This enhancement by cVA is lost when both males and females lack Or67d. Error bars represent SEM. Significant differences from the wildtype controls calculated using Student's $t$ test. For all bars, $n \geq 6$. ns, Not significant. ${ }^{*} p<0.05$, ${ }^{* *} p<0.01$.

males toward wild-type females. In fact, the courtship index of Or67 $d^{G A L 4}$ males paired with wild-type females in the presence of cVA was significantly higher than wild-type male controls (Fig. 3). This increase likely reflects the enhanced receptivity induced by cVA in the wild-type females, combined with loss of cVAmediated inhibition in the Or67d mutant males, as Or67d mutant males paired with Or67d mutant females are insensitive to the effects of cVA (Fig. 3). Together, these results provide strong evidence that T1 neuron activation is both necessary and sufficient to elicit sexually dimorphic cVA-induced mating behaviors.

\section{Discussion}

In this study, we isolated the sexually dimorphic behavioral effects of T1 neuron activation on male and female Drosophila courtship behavior. Using a dominant-active lush allele, lush ${ }^{D 118 A}$, we were able to increase the basal activity of the T1 neurons twofold in the absence of cVA. This increased firing rate was sufficient to reproduce sexually dimorphic behaviors that normally occur in the presence of cVA. Furthermore, the alterations in both male and female behavior due to $l u s h^{D 118 A}$ and cVA were both abrogated by loss of the downstream receptor Or67d. These findings indicate that activity in the T1 circuit alone is necessary and sufficient to mediate the effects of cVA on courtship behaviors.

Introduction of recombinant $\mathrm{LUSH}^{\mathrm{D} 118 \mathrm{~A}}$ into the sensillum lymph of $\mathrm{T} 1$ sensilla can stimulate $\mathrm{T} 1$ neurons from 1 to 15 spikes/s (Laughlin et al., 2008). Transgenic expression of $\mathrm{LUSH}^{\mathrm{D} 118 \mathrm{~A}}$ increased the basal firing rate of the T1 neurons approximately twofold in the transgenic animals, whereas there was no increase in the average $\mathrm{T} 2$ neuron firing rates. Thus, LUSH $^{\text {D118A }}$ specifically activates T1 neurons when expressed as a transgene regulated by the lush promoter. We suspect that this modest increase in firing compared with direct infusion of recombinant protein may reflect desensitization of the T1 neurons due to chronic LUSH ${ }^{\mathrm{D} 118 \mathrm{~A}}$ stimulation or perhaps a structural difference when LUSH $^{\text {D118A }}$ is expressed through the support cell secretory pathway. Indeed, it appears that LUSH $^{\text {D118A }}$ expressed in the fly is cVA-sensitive (supplemental Fig. S4, available at www.jneurosci.org as supplemental material) but not when recombinant bacterially expressed protein is infused via the recording pipette (Laughlin et al., 2008). Based on our observation of courtship behavior, this further increase in T1 firing rate by exogenous cVA has no additional effect on courtship behaviors of lush ${ }^{D 118 A}$ transgenic flies in which this circuit is already activated (Fig. 3). It is known from the work of Schlief and Wilson (2007) that even small increases in T1 firing rate can induce large increases in the frequency of downstream projection neuron firing. Indeed, the robust effects on courtship behavior in the LUSH $^{\mathrm{D} 118 \mathrm{~A}}$ lines support the idea that the T1 circuit is activated in these animals. This is consistent with cVA acting as an inhibitor of mating in males to avoid unproductive courtship with other males or possibly recently mated females, but as an aphrodisiac in females when they are being courted by a cVAproducing male.

There has been recent controversy over whether cVA-induced mating behaviors are mediated by Or67d, Or65a, or both (Ejima et al., 2007; Kurtovic et al., 2007). Our data support the notion that cVA acts exclusively through T1 neurons expressing Or67d, thus affecting courtship behavior. This is based on several observations. First, transgenic $l u s h^{D 118 A}$ specifically increases T1 activity and induces cVA-related behaviors. No effect was observed in T2 neurons. Second, the effects of $l u s h^{D 118 A}$ and cVA are both blocked by loss of Or67d, indicating that these behaviors are elicited through this pathway. Finally, in the presence of $\mathrm{LUSH}^{\mathrm{D} 118 \mathrm{~A}}$, cVA has no additional effects on behavior in these engineered flies. Together, these findings present a compelling argument for Or67d providing the major, if not exclusive, sensory pathway-mediating cVA behaviors. Our findings do not support those of Ejima et al. (2007), which found that tetanus toxin expressed under the Or65a promoter blocked cVA-induced suppression of naive male courtship, whereas expression under the Or67d promoter had no effect. It is possible that the ectopic tetanus toxin expression Ejima et al. (2007) noted in these lines produced additional effects on courtship behavior. Furthermore, activation of Or65a by cVA requires ligand concentrations far above the physiologically relevant range (van der Goes van Naters and Carlson, 2007; Laughlin et al., 2008), suggesting that cVA may not be a physiologic ligand for Or65a. Indeed, cVA was recently shown to mediate male-male aggression, and these behaviors also appear to require Or67d but not Or65a (Wang and Anderson, 2009). However, given our findings, we have no explanation for why tetanus toxin expressed by the Or67d promoterGal4 driver failed to inhibit male courtship (Ejima et al., 2007). Analysis of Or65a mutants in the future may shed light into these issues.

Our results confirm and extend the findings of Kurtovic et al. (2007), which demonstrated increased male-male courtship in males lacking Or67d and increased latency to mate in females lacking this receptor. These studies revealed that Or67d is important for sexually dimorphic, cVA-related courtship behaviors, but did not address whether activity in Or67d neurons alone accounts for all of these behaviors. Here we show that activating the T1 pathway with LUSH ${ }^{\mathrm{D} 118 \mathrm{~A}}$ has the opposite effects on behavior compared with the loss of function mutation in Or67d. 
Furthermore, we show that activation of T1 neurons enhances female receptivity to courting males. We conclude that the T1/ Or67d circuit is not only necessary but is sufficient for these behaviors.

Finally, it should be possible to identify sexual dimorphism in the downstream neurons of the T1 circuit. Indeed, the exclusive target of Or67 d sensory neurons is the DA1 glomerulus (Couto et al., 2005). Datta et al. (2008) recently discovered sexually dimorphic branches in the projection neurons that transmit activity in this glomerulus to higher brain centers. It remains to be seen whether these differences are truly responsible for the differences in behavior observed in male and female fruit flies. However, with the powerful genetic tools available in Drosophila (Pfeiffer et al., 2008), it should be possible to elucidate the complete neuronal map of this cVA-activated circuit.

\section{References}

Amrein H (2004) Pheromone perception and behavior in Drosophila. Curr Opin Neurobiol 14:435-442.

Benton R, Vannice KS, Vosshall LB (2007) An essential role for a CD36related receptor in pheromone detection in Drosophila. Nature 450:289-293.

Clyne P, Grant A, O'Connell R, Carlson JR (1997) Odorant response of individual sensilla on the Drosophila antenna. Invert Neurosci 3:127-135.

Couto A, Alenius M, Dickson BJ (2005) Molecular, anatomical, and functional organization of the Drosophila olfactory system. Curr Biol 15:1535-1547.

Datta SR, Vasconcelos ML, Ruta V, Luo S, Wong A, Demir E, Flores J, Balonze K, Dickson BJ, Axel R (2008) The Drosophila pheromone cVA activates a sexually dimorphic neural circuit. Nature 452:473-477.

Dickson BJ (2008) Wired for sex: the neurobiology of Drosophila mating decisions. Science 322:904-909.

Ejima A, Smith BP, Lucas C, van der Goes van Naters W, Miller CJ, Carlson JR, Levine JD, Griffith LC (2007) Generalization of courtship learning in Drosophila is mediated by cis-vaccenyl acetate. Curr Biol 17:599-605.
Greenspan RJ, Ferveur JF (2000) Courtship in Drosophila. Annu Rev Genet 34:205-232.

Ha TS, Smith DP (2006) A pheromone receptor mediates 11-cis-vaccenyl acetate-induced responses in Drosophila. J Neurosci 26:8727-8733.

Ha TS, Smith DP (2009) Odorant and pheromone receptors in insects. Front Cell Neurosci 3:10.

Hall JC (1994) The mating of a fly. Science 264:1702-1714.

Jin X, Ha TS, Smith DP (2008) SNMP is a signaling component required for pheromone sensitivity in Drosophila. Proc Natl Acad Sci U S A 105:10996-11001.

Kim MS, Repp A, Smith DP (1998) LUSH odorant-binding protein mediates chemosensory responses to alcohols in Drosophila melanogaster. Genetics 150:711-721.

Kurtovic A, Widmer A, Dickson BJ (2007) A single class of olfactory neurons mediates behavioural responses to a Drosophila sex pheromone. Nature 446:542-546.

Laughlin JD, Ha TS, Jones DN, Smith DP (2008) Activation of pheromonesensitive neurons is mediated by conformational activation of pheromonebinding protein. Cell 133:1255-1265.

Manoli DS, Meissner GW, Baker BS (2006) Blueprints for behavior: genetic specification of neural circuitry for innate behaviors. Trends Neurosci 29:444-451.

Pfeiffer BD, Jenett A, Hammonds AS, Ngo TT, Misra S, Murphy C, Scully A, Carlson JW, Wan KH, Laverty TR, Mungall C, Svirskas R, Kadonaga JT, Doe CQ, Eisen MB, Celniker SE, Rubin GM (2008) Tools for neuroanatomy and neurogenetics in Drosophila. Proc Natl Acad Sci US A 105:9715-9720.

Ronderos DS, Smith DP (2009) Diverse signaling mechanisms mediate volatile odorant detection in Drosophila. Fly (Austin) 3:290-297.

Schlief ML, Wilson RI (2007) Olfactory processing and behavior downstream from highly selective receptor neurons. Nat Neurosci 10:623-630.

van der Goes van Naters W, Carlson JR (2007) Receptors and neurons for fly odors in Drosophila. Curr Biol 17:606-612.

Wang L, Anderson DJ (2009) Identification of an aggression-promoting pheromone and its receptor neurons in Drosophila. Nature 463:227-231.

Xu P, Atkinson R, Jones DN, Smith DP (2005) Drosophila OBP LUSH is required for activity of pheromone-sensitive neurons. Neuron 45:193200. 